\title{
Nonlinearity and Energy Conservation: A Possible Way to Harness Energy from the Subquantum Medium
}

\author{
J.R. Croca ${ }^{1,2}$, P. Castro ${ }^{2}$, R. Moreira ${ }^{2}$ and M. M. Silva ${ }^{2}$ \\ ${ }^{1}$ University of Lisbon, Faculty of Sciences, Department of Physics \\ ${ }^{2}$ Center of Philosophy of Sciences of the University of Lisbon \\ Email: jncroca@fc.ul.pt; jpcastro@fc.ul.pt; ranmoreira@gmail.com; mmcsilva@fc.ul.pt
}

\begin{abstract}
In some situations nonlinearity seems to pervade in nature. Indeed, in adequate conditions, a minor action may give origin to a very large reaction, only apparently providing a gain in output power from a lesser inputted value. Extending de Broglie seminal ideas to a more general pilot-wave theory, we describe the relation between nonlinearity and energy conservation, dismissing any apparent contradiction between the two. We also present what seems to be a possible way to harness energy from the pervading subquantum medium.
\end{abstract}

Keywords: Energy conservation, subquantum medium, nonlinearity, pilot wave theory, nonlinear induction principle.

\section{Nonlinearity in the context of a Pilot-Wave Theory}

Even if the great French physicist Louis de Broglie [1] explained the dualism particle-wave mystery in a natural causal way, his ideas were so daring that only a minority of physicists understand their deep meaning at the time. One of his fundamental proposals was to consider that each atomic entity is a real objective physical complex system with a corpuscle, surrounded by a quantum wave. This wave is not an elusive, metaphysical probability wave, as claimed by orthodox quantum mechanics [2], but a real undulatory perturbation, propagating in a subquantum medium and guiding the corpuscle along its trajectory [3]. This piloting or guiding effect may be seen as an information transferring process, where a subtle entity, the pilot-wave, with lesser energetic content, guides the movement of the corpuscle, a much more energetic system in itself. The wave seems to accomplish such a feat in a sophisticated way, without the usual plain mechanistic pushes. This new and somehow revolutionary view, once developed to its natural consequences [4], implies taking a critical stand towards the linear Cartesian method and its main claim that the whole equals the sum of the parts. The parts, are classically assumed to combine in such a way that no matter the interacting process, each part maintains its own individuality. In this sense, the action-reaction law is no more than a simple consequence of this atomistic and individualist stance.

Independently of what de Broglie proposed, the nowadays linear and Cartesian way of thinking is, by itself, quite surprising. How can one look at Nature without understanding that, at least in some observable physical situations, one is witnessing nonlinear complex phenomena, in which a small action gives rise to a huge reaction. Take for instance the case of electromagnetism. An electric current flowing in a circular isolated conductor produces a magnetic field. This magnetic field intensity is proportional to the number of circular coils and to the input current. In these conditions, if one places two similar devices together, a repulsive or attractive force is expected to be observed, as shown in Fig.1.

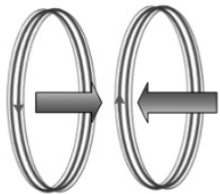

Figure 1. Opposite magnetic forces, indicated by arrows. Currents flowing in opposite directions along the two circuits should produce a repulsion. 
Still, and here is the crucial point, unless the current flowing in the coils is very intense, as experimental evidence shows, the attractive or repulsive force is so insignificant, that practically no motion of the coils is observed.

To circumvent these concrete experimental facts, Ampere, Faraday and others came out with the providential idea of wrapping the isolated conductors around a massive iron core, as shown in Fig.2.

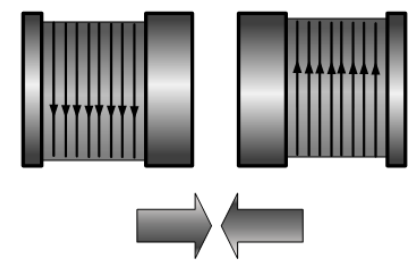

Figure 2. Electromagnets.

In this case, for the very same input current, flowing along the same number of coils, a strong repulsive force can now be observed as the two devices repel each other.

What did just happen, providing an outcome so startling different from the one in the previous situation? Clearly, a small action, due to the organized flow of electric currents running through the wires, triggered the chaotic circular micro-currents of the iron core, also guiding these. That is, inducing them to follow an averagely preferential direction. This same very action imprinted on a core made of wood, plastic or other similar substances has no visible effect. One could thus say that such materials are not sensitive to the organizing information carried by the electric current flowing in the conductors. Naturally, this effect is usually explained dismissing the nonlinear nature of the phenomena, as here stated.

In the present article we will suggest a possible way of orienting, piloting or guiding the random motion of free electrons in a conductor, so that a net electric current can be produced by a nonlinear process and, furthermore, used to deliver useful work.

Still, it must be said that we do not wish to deny the great merits of classical physics, rooted mainly on the linear Cartesian approach. What is at stake is that if one wants to go further towards a better understanding of Nature, it is necessary to embrace and develop a complex nonlinear physics containing the linear approach as a particular case [5].

In what follows we will apply a pilot-wave model inspired in the one proposed by de Broglie [4]. In such conditions, it will be assumed that instead of metaphysical probability potential quantum entities, corpuscles and its waves do have physical reality, independently of the observational powers of human beings. Furthermore, we shall accept that these subquantum waves are finite perturbations in space and time, fairly described by Morlet wavelets $[5,6]$.

It should be mentioned that a Morlet wavelet will reduce to a harmonic plane wave, once the sigma $\sigma$ parameter, representing the wavelet spatial extension, is very large. That is, whenever $\sigma \rightarrow \infty$, one obtains:

$$
\theta=A e^{-\frac{(\mathrm{x}-\mathrm{vt})^{2}}{2 \sigma_{0 \mathrm{x}}^{2}}+\mathrm{i}(\mathrm{kx}-\omega \mathrm{t})} \underset{\sigma \rightarrow \infty}{\longrightarrow} \theta=A \mathrm{e}^{\mathrm{i}(\mathrm{kx}-\omega \mathrm{t})}
$$

The last expression on the right side is, in fact, the usual mathematical expression for a harmonic plane wave, spreading infinitely over space and time, which is derived from the abstract case resulting from the more general and realistic wavelet expression, on the left side of (1).

The second feature one should have in mind is that the wavelet expression is formally a solution of a non linear differential equation, called the Master equation [4], reading:

$$
-\frac{\hbar^{2}}{2 \mu} \nabla^{2} \theta+\frac{\hbar^{2}}{2 \mu} \frac{\nabla^{2}\left(\theta \theta^{*}\right)^{1 / 2}}{\left(\theta \theta^{*}\right)^{1 / 2}} \theta+\mathrm{V} \theta=i \hbar \theta_{\mathrm{t}}
$$

where $\hbar$ is the Planck constant, and $\mu$ represents the particle's mass. It should be noted that whenever the non linear term, the second term on the left, is null, one obtains Schrödinger's linear differential equation. 
Finally, the wavelet has determined temporal frequency $\omega$ and spatial frequency $k$ at all times. This has major consequences to what is to be thought about the nature of physical systems at quantum scales. In fact, a set of generalized uncertainty relations can be obtained using wavelet composition, following a procedure very much analogous to the one introduced by Niels Borh in his famous 1927 Como lecture paper $[7,8]$.

The generalized uncertainty relations can be written as:

$$
\begin{gathered}
\Delta p_{x}=\frac{\hbar}{\Delta x} \sqrt{1-\Delta x^{2} / \sigma_{0 x}^{2}} \\
\Delta E=\frac{\hbar}{\Delta t} \sqrt{1-\Delta t^{2} / \sigma_{0 t}^{2}}
\end{gathered}
$$

For these expressions we have considered $\sigma_{0 x}=v \sigma_{0 t}$ in the second relation, where $\sigma_{0 x}$ represents the spatial extension of the mother wavelet used in the wavelet packaging and $v$ is the wavelet average velocity.

Once more, whenever $\sigma \rightarrow \infty$, one obtains the usual Schrodinger's uncertainty relations.

The herein model is therefore formally more general than traditional orthodox quantum mechanics. From a mathematical point of view, it contains the Fourier formalism as a particular case. It should be mentioned that de Broglie's original double solution theory $[9,10]$ used harmonic infinite waves to describe real subquantum perturbations, piloting corpuscles. Our model also assumes the plain existence of such waves, using wavelets instead to describe finite subquantum perturbations. Thus we are introducing yet another element of realism, as there are no infinite perturbations, while adopting a more general mathematical framework.

Indeed, we will assume that each corpuscular system has its own overall associated subquantum wave, a finite perturbation propagating trough space. The motion of the complex corpuscle will be such, that, in average, it will tend to move to the regions where the intensity density of the guiding wave is greater. This statement, dubbed the Principle of Eurhythmy, constitutes an equivalent formulation to the Principle of Guidage, used by de Broglie [4,5,10]. We call our model Eurhythmic Physics [5].

Finally, and before applying the model to the induction phenomena, we will accept without any formal justification that the electromagnetic field must in some way result from the overall contribution of subquantum waves. Once we hypostasize for the reality of electronic finite waves, it is a matter of common sense that these cannot be physically disconnected from the overall resulting EM field. For the purposes of this paper, the EM field will thus be thought as an operational field, representing a particular composition of subquantum waves.

Even in the context of a pilot-wave model such as the one herein presented, the occurrence of a nonlinear interaction, where a large amount of particles are acted upon by the same subquantum wave, may apparently lead to Energy conservation violation. In what follows it will be reasoned that such situation can be adequately dismissed once three major basic assumptions are additionally accepted. Namely:

1. That all systems in Nature draw their energy from the pervading subquantum medium, by means of complex nonlinear oscillatory process. The overall energy used by the systems plus the subquantum internal energy remaining constant.

2. That each physical system has its proper own energy. The capture energy efficiency of a system depends on its particular structural relational properties and on the frequency of the oscillatory process.

3. That all interactions among complex physical systems are mediated by subquantum waves. These waves being produced, emitted and received by each interacting system.

We will thus accept that when a given physical system is under the influence of a propagating wave, the frequency of the wave induces the system to oscillate coherently. And it will be by means of this complex oscillatory process that the system will draw energy from the subquantum medium.

It will also be assumed that each complex physical system retrieves energy from the subquantum medium, accordingly to the general phenomenological quantum mechanical relation:

$$
E_{s}=h_{s} v
$$


where $E_{s}$ stands for the amount of energy drawn from the subquantum medium by the complex system $S$, $v$ is the frequency of the oscilatory process by which the gathering process occurs and $h_{s}$ measures the system's overall efficiency in doing so. According to the second assumption, the value of $h_{s}$ in general will be different for each complex system. Still, for the same kind of systems, at some scale, such value may averagely be considered the same. This would be, for instance, the case of Plank's constant, applicable to atomic systems, that is, where $h_{s}=h$.

Expression (5) is assumed to have a general scope and therefore may be adequately used to describe the behavior of different physical complex systems, at different scales of observation and description of Reality. As a natural consequence, the nonlinear complex wave interaction model is extended to other physical domains besides the quantum. In fact, such an approach has already been applied successfully to the Solar system, explaining the Titius-Bode regularity [11] and in addition providing a value for a parameter similar to $h_{s}$.

Finally, it should be mentioned that assumption 3 implies that although a subquantum wave will still carry some energy, its amount will be negligible compared with the energy drawn by emitting and receiving systems [12]. In short, a subquantum wave acts like an information carrier between corpuscular relatively localized systems. The wave uses a minimum amount of energy (also drawn from the subquantum medium) to influence the oscillatory regimes by which the corpuscles capture energy from the subquantum medium.

We define an oscillatory regime as the frequency at which a given structure is able to oscillate, drawing energy from the subquantum medium, accordingly to expression (5). This same expression defines mathematically what is to be understood by such regime. It is expected that each system can assume any one of a characteristic set of oscillatory frequencies.

\section{The Nonlinear Induction Principle}

After hard experimental work Faraday found out that when an electric current produced by a Volta pile flows in a primary conductor, a similar current appears in a nearby positioned secondary conductor, only if the intensity of the applied current changes in time. In the context of Eurhythmic Physics [5], the electrical potential applied to the primary corresponds, in fact, to a very asymmetrical imposed pilot wave field. The electrons sense this field and, according to the Principle of Eurhythmy, tend to move in the direction of the field's higher intensity density zone. When they finally reach that zone, they tend to move randomly inside it, with a null observable average velocity. In order to obtain a new current flow, now in the opposite direction, one has to invert the pilot wave field asymmetry. This corresponds, of course, to an alternated electrical potential field, producing an alternated current.

The electric flow of electrons along the primary conductor reinforces the external applied theta wave field, making visible what is traditionally identified as a magnetic field, originating in the conductor. The overall pilot wave field intensity surrounds radially the coil, as sketched in Fig.3.

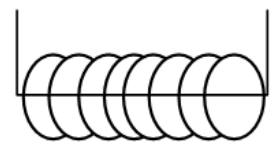

Figure 3. The motion of the electrons in the conductor produces a surrounding field

This surrounding field decreases with the distance from the wire in an approximately Gaussian way, as indicated in Fig.4.

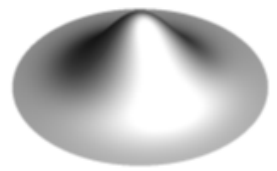

Figure 4. Radial electromagnetic theta wave field intensity density $I_{M}$ 
However, as have been shown elsewhere [5], not very close, nor very far from the origin, the decreasing rate of the intensity field density may be fairly described by the inverse square of the distance,

$$
I_{M} \propto 1 / r^{2}
$$

In such circumstances, if one places a coil in the proximal vicinity of the primary, the field involves also the secondary, as shown in Fig.5.

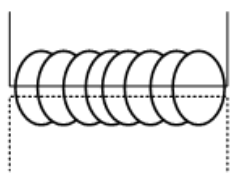

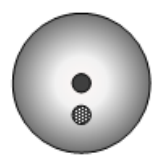

b

Figure 5. An independent conductor, placed in the vicinity of the first, feels the same field: a) longitudinal view, b) transversal section.

For an input changing in time, an induced similar current is observed in the secondary circuit. In normal conditions, the free electrons in the secondary move incessantly in a random way. However, when they receive the information from the field generated by the primary, they start moving along a preferential direction in the secondary coil. They do this in a way analogous to what we have already described in the case of the iron core. Here again, a minor action induces the chaotic motion of the electrons to follow a preferable direction, thus producing an observable induced electric current in the secondary, Fig.6.

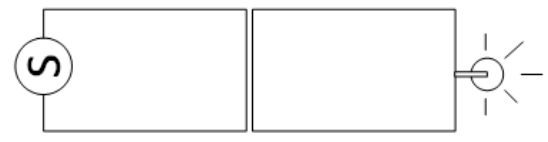

Figure 6. Induced current in the secondary.

Now, and this is a very important point, one can place more than one secondary in the vicinity of the field, generated in the primary, as indicated in Fig.7.

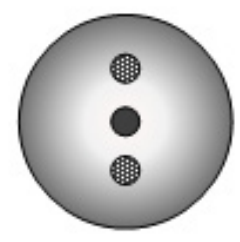

Figure 7. Transversal section of the field of the primary with two secondary conductors.

And since we are assuming that induction is a nonlinear phenomenon, where the wave information carrier is the meaningful factor, the induced current is expected to be nearly the same in the two outputs.

At this point we should mention that we believe this multiplication induction effect to be a sound working hypothesis. Although it still lacks commonly acceptable empirical confirmation, we also believe that given our interpretation of the pilot wave as a nonlinear agency, it is a research line worth pursuing.

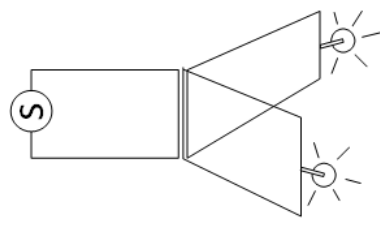

Figure 8. One input and two similar outputs. 
Our work here is mostly devoted to the fact that such theorization does not imply energy conservation violation.

As may be hypostasized, according to what may be called a nonlinear organizing principle, it would then be possible to place more than two output secondary circuits in the vicinity of the primary and, for the same input, within certain conditions, have similar or possibly higher overall power outputs.

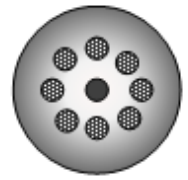

Figure 9. Transversal section. One input and eight similar outputs.

Acknowledging the nonlinear effect of a piloting or guiding subquantum field, we would, for the sake of argument, be able to obtain a so-called over-unity generator. That is, a device that would be wrongly credited to produce more energy than the one it consumes. In reality, even if we were to measure a higher output power in the secondary circuits, we truly wouldn't be before an over-unity device. In fact, the principle of energy conservation wouldn't have been surpassed. What happens is that due to the coherent behavior of electrons in the secondary circuits, the overall efficiency with which energy is being gathered from the subquantum medium, would be higher than the one corresponding to the primary circuit. That is, the value $h_{2}$ for the secondary circuits, taken as a whole subsystem, would be higher than the $h_{1}$ value for the primary circuit.

Using relation (5), this would imply an output energy $E_{2}$ in the secondary circuits higher than the input energy $E_{1}$ in the primary circuit. However, recalling the first assumption, once we take into account the subquantum medium, we would come to the fact that the energy drawn by both interacting systems, the primary and secondary circuits, plus the remaining energy in the subquantum medium would be constant. That is, the overall energy would have been conserved.

The most common situation occurs when $h_{1} \geqslant h_{2}$, where the igniting system uses more energy than the energy provided by the ignited system, a situation usually characterized saying that there is energy loss. However, it must be stressed that the theoretical possibility for what can now be called pseudo overunity devices, by means of a nonlinear interaction occurring among its parts, becomes very real. All we must do is to find the appropriate geometric disposition of the primary and secondary conductors, making more efficient the organizing effect of the applied EM field (the piloting wave field) on all conductors.

Again, it should be mentioned that no energy is being created. Indeed, we are only taking advantage of the energy already available (and drawn from the subquantum medium) by the free electrons. In most normal situations they have an incessant random motion. However, in adequate interacting conditions, the nonlinear stimulus of the subquantum organizing field in the primary makes most of the free electrons start moving averagely in the same direction, producing a net flow of electric current.

The parameter $h_{s}$ in (5) can therefore also be understood as a measure of the state of organization of a system, after being stimulated by an information carrier. In this case, the information carrier is the applied electromagnetic field and, by the second assumption, a very important part of that information will be the applied field frequency.

From what was said previously, we will now suggest the Nonlinear Induction Principle, stating as follows.

Given a primary circuit $S_{1}$, generating an inducing electromagnetic field, using a certain amount of energy $E_{1}$, and given a set of two or more secondary circuits $S_{2}$ in the near vicinity of the applied field, depending on the spatial disposition of the conductors, on the geometry of the field and on its frequency $v$, two situations may occur:

a) If $h_{1} \geqslant h_{2}$, we will have $E_{1} \geqslant E_{2}$ defining a unity transformer.

b) If $h_{1}<h_{2}$, we will have $E_{1}<E_{2}$ defining a pseudo over-unity transformer.

At this point, it must be said that over the years, several inventors have announced machines able to extract energy from the surrounding medium or able to provide energy in an auto sustained manner. What indeed would be called perpetual machines of the first kind? As stated before, without taking into account the subquantum medium energy contribution, the behavior of such machines would contradict 
the principle of conservation of energy, and therefore be impossible. However, taking into account the Nonlinear Induction Principle, although some of such allegations can be dismissible due to errors made by their authors, it is also conceivable that some of them may have in fact corresponded to situations where a pseudo over-unity device was at work.

\section{Conclusion}

Using theoretical arguments based upon a pilot wave theory applied to electromagnetic phenomena, we have described a possible way to harness energy from the subquantum medium. A new phenomenological approach to physical phenomena that involves taking into account the nonlinear effect a piloting wave field may have over corpuscles. We have also suggested three major assumptions that in short assume subquantum waves as information carriers between systems, thus defining the nature of the interactions between them. In the same spirit, we have proposed the Nonlinear Induction Principle, an extension of Farady's law, dismissing any contradiction between nonlinear interactions and the Principle of energy conservation.

Acknowledgements. This work is funded by FCT (Portugal) through CFCUL (project UID/FIL/00678/2013).

\section{References}

1. L. de Broglie, Non-Linear Wave Mechanics: a Causal Interpretation, (Elsevier, Amsterdam, 1960); L. de Broglie, The Current Interpretation of the Wave Mechanics, A critical study, (Elsevier, Amsterdam, 1964).

2. M. Jammer, The Conceptual development of Quantum mechanics, Mc-Graw-Hill, London, 1966.

3. C. Dewdney. Particle trajectories and interference in a time-dependent model of neutron single crystal interferometry. Physics Letters A, 109(8):377-384, 1985.

4. J.R. Croca, Towards a nonlinear quantum physics, World Scientific, London, 2003.

5. J.R. Croca, Eurhythmic Physics, or Hyperphysics, The Unification of Physics, Lambert Academic Publishing, Berlin, 2015.

6. A. Grossmann and J. Morlet, SIAM J. Math. Anal. 3, 723(1989); Decomposition of Function into Wavelets of Constant Shape and Related Transforms, (World Scientific, Singapore, 1980); C.K Chui, An Introduction to Wavelets, (Academic Press, N.Y. 1992); B.B. Hubbard, The Word According to Wavelets, (A. K. Peters Wellesley, Massachusetts.

7. Castro, P., Croca, J. R., Gatta, M. \& Moreira, R. (2017) Generalized Uncertainty Relations in Quantum Mechanics and the Principles of Completeness in Physics, Physical Science International Journal, Vol.: 16, Issue.: 4.

8. Bohr, N. Como Lectures (1928), Collected Works, Vol. 6, (North-Holland, Amsterdam, 1985).

9. Louis de Broglie, "La mécanique ondulatoire et la structure atomique de la matière et du rayonnement" em Le Journal de Physique et le Radium (6), 8, 225-41, (Paris: 1927).

10. Guido Bacciagaluppi e Antony Valentini, Quantum Theory at the Crossroads: Reconsidering the 1927 Solvay Conference. (Reprint edition) (New York: Cambridge University Press, 2013).

11. J.R. Croca, P. Castro, M. Gatta, A. Cardoso and R. Moreira, Pilot-wave gravity and the Titius-Bode Law, accepted in New Horizons in Mathematical Physics 2017.

12. J.R. Croca, De Broglie Tired Light Model and the Reality of the Quantum Waves, Foundations of Physics, Vol 34, n⿳⺈ 12, pags.1929-1954, 2004. 